\title{
Erratum to: Simple steps to develop trial follow-up procedures
}

Ona McCarthy ${ }^{1 *}$, Rebecca S. French², lan Roberts ${ }^{1}$ and Caroline Free ${ }^{1}$

\section{Erratum}

Unfortunately, the original version of this article [1] contained an error. There were errors in the reference numbers in Additional file 1. This has now been corrected and Additional file 1 is included here with the correct reference numbers.

\section{Additional file}

Additional file 1: Key findings and implications for follow-up design. Details of follow-up strategies where there is evidence of increasing the odds of response. (DOCX $19 \mathrm{~kb}$ )

\section{Author details}

'Department of Population Health, London School of Hygiene and Tropical Medicine, Keppel St, London WC1E 7HT, UK. ${ }^{2}$ Department of Social and Environmental Health Research, London School of Hygiene and Tropical Medicine, 15-17 Tavistock Place, London WC1H 9SH, UK.

Received: 1 June 2016 Accepted: 6 September 2016

Published online: 06 October 2016

\section{Reference}

1. McCarthy O, French RS, Roberts I, Free C. Simple steps to develop trial

follow-up procedures. Trials. 2016;17:28.

\footnotetext{
* Correspondence: ona.mccarthy@lshtm.ac.uk

'Department of Population Health, London School of Hygiene and Tropical Medicine, Keppel St, London WC1E 7HT, UK

Full list of author information is available at the end of the article
} 